\title{
Novelties in Brazilian Waltheria L. (Byttnerioideae, Malvaceae): two new species and one re-establishment
}

\author{
Thales Silva Coutinho ${ }^{1 *}$ (D), Matheus Colli-Silva ${ }^{2}$ (1) and Marccus Alves ${ }^{3}$ (D)
}

Received: March 10, 2020

Accepted: May 29, 2020

\begin{abstract}
This work validates two Waltheria species endemic to campo rupestre with complete descriptions, illustrations, updated geographical distributions and comments on taxonomic affinities. Waltheria biribiriensis and Waltheria terminans are restricted to the Southern Espinhaço Province in eastern South America, occurring on mountain tops where campo rupestre vegetation prevails. Furthermore, Waltheria brachypetala, a species of dry deciduous forests in Brazil that was long considered as conspecific with $W$. ferruginea due to its shrubby lifeform and similar indumentum, is re-established based on differences of phyllotaxy, blade shape and inflorescence type. The first occurrence records of $W$. brachypetala in the state of Paraíba are also provided, as well as the first record in the Atlantic Forest domain. Lastly, a lectotype is designated for W. ferruginea.
\end{abstract}

Keywords: campo rupestre, endangered species, Hermannieae, heterostily, Malvales, re-establishment, vulnerable species

\section{Introduction}

Waltheria is a pantropical genus including c. 50-60 species (Bayer \& Kubitzki 2005), with 23 of them native to Brazil (Silva-Coutinho et al. 2019). Although the genus is widespread, species of Waltheria are usually narrowly restricted to particular regions of the Neotropics, northern Mexico and North America (Saunders 1993). Most species are endemic to the open, seasonally dry habitats in South America, namely the Brazilian Cerrado, Caatinga and Chaco (Saunders 1995, unpublished).

Waltheria is a shrubby-herbaceous genus belonging to Byttnerioideae -one of the nine clades in Malvaceae sensu
Alverson et al. (1999), supported by further studies (e.g. Whitlock et al. 2011; Richardson et al. 2015). Byttnerioideae is characterized by having clawed and often caducous petals, small flowers, shorter than $2-3 \mathrm{~cm}$ in length, without epicalyx and usually with a reduced or absent androgynophore. Palynological traits can be diagnostic for the subfamily as well, and most species exhibit elongated pollen grains with prominent operculate apertures (Cristóbal 1968; Bayer \& Kubitzki 2005). Waltheria is placed in Hermannieae, a tribe distinguished by having flat petals and absence of androgynophore (Saunders 1995; unpublished). Waltheria is also within subtribe Melochinae, where trends such as the gradual reduction

1 Programa de Pós-graduação em Biologia Vegetal, Departamento de Botânica, Centro de Biociências, Universidade Federal de Pernambuco, 50670-901, Recife, PE, Brazil

2 Departamento de Botânica, Instituto de Biociências, Universidade de São Paulo, 05508-090, São Paulo, SP, Brazil

3 Departamento de Botânica, Centro de Biociências, Universidade Federal de Pernambuco, 50670-901, Recife, Pernambuco, Brazil

* Corresponding author: thales_scoutinho@hotmail.com 
of the number of ovules per locule are observed (Saunders 1995, unpublished; Bayer \& Kubitzki 2005).

Melochinae species usually have heterostylic flowers, where two floral morphotypes, "longistylous" and "brevistylous", exhibit different stamen/style length ratios (Kohler 1976; Saunders 1993). Waltheria presents the floral polymorphism typical of the subtribe as well as the uttermost case of carpel reduction, with its species bearing only one carpel with one locule and two ovules per locule, typically producing only one seed per capsular fruit (Saunders 1995, unpublished; Bayer \& Kubitzki 2005).

Several infrageneric classifications have been previously applied to Waltheria (e.g. Schumann 1886; Rose 1899; Saunders 1995, unpublished). Saunders (1995, unpublished) provided one of the most important contributions to the systematics of the genus, addressing evolutionary aspects of heterostyly as well as infraspecific taxonomical circumscriptions (except for the W. indica complex). Saunders described ten new species (1995, unpublished), with only three of them effectively published (Saunders 2005; Saunders \& Pozner 2007).

We have identified unpublished names of taxa endemic to specific regions of campo rupestre vegetation that require revision. According to Saunders (1995, unpublished), the areas of campo rupestre in the eastern South American mountaintops are the major center of species richness of Waltheria, with many species or potential clades, such as the Waltheria ferruginea alliance sensu Saunders (1995, unpublished), restricted to this high-elevation habitat. These species are found in different areas of campo rupestre determined as the newly recognized districts of the Southern Espinhaço province (Colli-Silva et al. 2019).

Given this background, we hereby present taxonomical and nomenclatural novelties in a set of species that belong in the informal Waltheria ferruginea alliance sensu Saunders (1995, unpublished). We provide detailed morphological information, updated distribution data and highlight morphological features that distinguish the different species in the alliance. Furthermore, we review the circumscription of the most widespread species of the alliance, Waltheria brachypetala, and its related taxon, $W$. ferruginea, providing detailed descriptions and a brief nomenclatural history, as both species were traditionally treated as conspecific, and data on biogeography and ecology.

\section{Materials and methods}

Specimens from the following collections were examined: ASE, BHCB, BR ${ }^{* *}$, CTES, EAC, ESA, $F^{* *}, G^{* *}, \mathrm{GH}^{* *}, \mathrm{HCDAL}$, HST $^{*}$, HUEFS $^{* *}$, HUVA* ${ }^{*}$ HVASF, JPB, K* ${ }^{* *}$, KW$^{* *}$, LE$^{* *}$, MAC, MBM, MO**, MPU**, MUFAL*, NY**, $\mathrm{P}^{* *}$, PEUFR, R, RB, SI, SP, SPF, UEC, UFP, UFRN (Thiers 2019, continuously updated; *not indexed; ${ }^{* *}$ photos). Protologues of Waltheria brachypetala and $W$. ferruginea were assessed. All examined vouchers were cited, except for $W$. brachypetala, where we selected specimens representing all states and types of vegetation where it occurs and its morphological variability.

Morphological terminology follows Webster et al. (1996) and Harris \& Harris (2001) for trichomes and indumentum, respectively; Radford et al. (1974) for leaf shape, and Weberling (1992) for inflorescence. We adopted the term "bracteole" to the foliaceous structures associated with the flowers, previously called "bracts" by Coutinho \& Alves (2019; 2020), Silva-Coutinho et al. (2019) and Saunders (1995, unpublished). Stigma terminology is based on Saunders (1993). Distribution maps were built using the software QGIS (https://www.qgis.org/en/site/), and preliminary conservation assessment was performed using the "ConR" package v. 1.2.4 in R (Dauby et al. 2017; R Development Core Team 2017).

\section{Results}

\section{Taxonomic treatment}

Waltheria ferruginea A. St-Hil. was described based on the collection Saint-Hilaire s.n., from Minas Gerais. He presented a diagnosis with a complete description of the species and a detailed illustration. Thirty-three years later, Waltheria brachypetala Turcz. was published based on the collection Blanchet 2744 from the state of Bahia (Turczaninow 1858). In the Flora Brasiliensis, Schumann (1886) proposed to synonymize the latter name under $W$. ferruginea, as he believed they were conspecific due to the lack of characters supporting their distinction.

Later on, Saunders (1995, unpublished) proposed the reestablishment of $W$. brachypetala, listing a set of characters supporting this distinction but never effectively publishing it. Esteves (2010) listed 26 species of Waltheria occurring in Brazil, considering W. brachypetala and W. ferruginea as distinct entities, but not presenting arguments to support her decision. According to Esteves (2010), W. brachypetala occurs in the dry, deciduous Caatinga in Northeastern Brazil (i.e. states of Bahia, Ceará, Pernambuco and Piauí), whereas $W$. ferruginea is restricted to the Cerrado and campo rupestre vegetations in the state of Minas Gerais. Consequently, $W$. brachypetala and $W$. ferruginea are now treated as different species in online databases and in herbarium collections.

1. Waltheria brachypetala Turcz. Bull. Soc. Imp. Naturalistes Moscou 31(1): 215. 1858. Type: BRAZIL. Bahia: Utinga, La Serra Acurua, 1858, Blanchet 2744 (Holotype: KW [photo]!; isotypes: BR [photo]!, F [photo]!, G [photo]!, GH [photo]!, K [photo]!, LE [photo]!, MO [photo]!, P [photo]!).

Shrubs 1.2-3.0 m tall. Branches erect, terete, slightly angulate apically, canescent, trichomes stalked, multiradiate, yellowish, c. $0.3 \mathrm{~mm}$ long; bark reddish-brown when dry, resinous, not lenticellate. Stipules 7.0-11.0 $\times 0.6 \mathrm{~mm}$, linear triangular, base truncate, apex acute, trichomes stalked multirradiate and sessile stellate, veins inconspicuous. 
Leaves simple, alternate, distichally arranged throughout the branches; petiole $0.60-1.10 \times 0.13-0.14 \mathrm{~cm}$, angulate, not canaliculate, tomentose; blade leaf chartaceous, concolorous, rarely discolorous, $4.2-10.2 \times 1.7-4.0 \mathrm{~cm}$, conduplicate or plane, lanceolate, base cordate to rounded, margins irregularly dentate throughout, teeth $0.8-1.8 \times 1.5-2.0$ $\mathrm{mm}$, apex acute, adaxial surface pubescent, trichomes sessile, stellate, abaxial surface canescent, trichomes sessile stellate and stalked multiradiate; venation actinodromous, 2 basal veins and 14-17 pairs of secondary veins, impressed adaxially, prominent abaxially. Inflorescence cymose, axillary along the branches, pedunculate, many-flowered; bracts 2-3-lobed, 6.0-8.0 mm long; peduncle $0.3-2.2 \mathrm{~cm}$ long, tomentose. Flowers distylous, sessile; bracteoles 4, 6.0-7.2 $\times 1.8-3.2 \mathrm{~mm}$, distinct or basally fused for $1.8-2.2 \mathrm{~mm}$, elliptic to widely elliptic, apex acute, entire to $2-3$-dentate apically, tomentose on both surfaces, trichomes sessile stellate on both surfaces, multinervate. Calyx 5-merous, gamosepalous, 7-7.2 × 2.7-3.0 mm, tubular-campanulate, 10-ribbed, externally pubescent, trichomes sessile, stellate, internally pubescent c. $2.0 \mathrm{~mm}$ long above base, tomentose on lobes, trichomes sessile, stellate, lobes c. $2.0 \times 2.0 \mathrm{~mm}$, apex acute; nectary c. $0.3 \mathrm{~mm}$ long, on the base of the internal surface. Corolla 5-merous, dialypetalous, yellow, petals adnate to the staminal tube for c. $0.3 \mathrm{~mm}, 7.0-7.5$ $\times 2.3-2.5 \mathrm{~mm}$, spatulate, both surfaces glabrous, apex emarginate, glabrous. Stamens 5 , fully or partially connate into a staminal tube, papillose apically, dithecae, thecae parallel, dehiscence rimose; gynoecium apocarpous, carpel one and locule one, ovary obovoid, sericeous, trichomes simple and 2-3-radiate, style one, lateral, tomentose, trichomes sessile, stellate, stigma one, elongate-plumose. Longistylous form: stamens $4.2-5.0 \mathrm{~mm}$ long, staminal tube 3.0-3.4 mm long, free portion of the filaments $0.3-0.6$ $\mathrm{mm}$ long, anthers 1.1-1.2 mm long, gynoecium 7.5-8.2 mm long, ovary $1.3-2.0 \times 0.8-0.9 \mathrm{~mm}$, style c. $3.8-5.0 \mathrm{~mm}$ long, tortuose, stigma 1.0-1.2 $\times 0.3 \mathrm{~mm}$. Brevistylous form: stamens $7.0-7.2 \mathrm{~mm}$ long, staminal tube c. $2.0 \times$ $1.0 \mathrm{~mm}$, free filaments c. $4.1 \mathrm{~mm}$ long, anthers c. $1.3 \mathrm{~mm}$ long, gynoecium c. $3.2 \mathrm{~mm}$ long, ovary $1.8 \times 0.7 \mathrm{~mm}$, style c. $1.5 \mathrm{~mm}$ long, stigma c. $1.0 \times 0.3 \mathrm{~mm}$ long. Capsule one, $2.8-3.5 \times 2.5 \mathrm{~mm}$, obovoid, chartaceous at the apex, then membranaceous below, apex truncate-rounded, pilose, trichomes stellate, concentrated on the apex, dehiscence loculicidal; seed one, 2.5-3.2 2 2 mm, obovoid, brown, glabrous, apex rounded.

Distribution and Habitat-Waltheria brachypetala is endemic to Brazil, where it is widely distributed in the Northeastern region (Alagoas, Bahia Ceará, Paraíba, Pernambuco, Piauí, Rio Grande do Norte, and possibly Sergipe, but without confirmed records), as well as in the state of Minas Gerais, in Southeastern Brazil. It occurs in open, dry habitats of the Caatinga, Cerrado and Atlantic Forest domains, in elevations between 30-718 m (Fig. 1).
We provide the first occurrence record in the state of Paraíba, as well as the first record in the Atlantic Forest domain.

Phenology - Waltheria brachypetala can be found with flowers and fruits throughout the year.

Conservation status-Due to its wide distribution range, Waltheria brachypetala is classified as Least Concern (LC) or Near Threatened (NT) according to our preliminary conservation assessment. This species occurs in protected areas in the states of Bahia (Raso da Catarina Ecological Station, Boqueirão da Onça National Park, Canudos Biological Station and Lagoa de Itaparica Environmental Protection Area), Ceará (Aiuaba Ecological Station, Serra das Almas Private Reserve of Natural Heritage and Ubajara National Park), and Pernambuco (Catimbau National Park), and in Grande Sertão Veredas National Park in the states of Bahia and Minas Gerais. Waltheria brachypetala also occurs on roadsides.

Comments-Schumann (1886) considered Waltheria brachypetala as conspecific to $W$. ferruginea, probably based on the following characters shared between both taxa: (1) shrubby lifeform, (2) leaves distichally arranged along the branches, (3) indumentum composed of stalked and multirradiate trichomes on branches and leaves, (4) axillary inflorescences, (5) similar bracteole shape, (6) distylous flowers and (7) capsule and seed shape. However, $W$. brachypetala differs from $W$. ferruginea especially due to: (1) stipules 7.0-11 mm long (vs. 3.0-3.2 mm long), (2) leaf blades lanceolate and conduplicate or plane (vs. elliptic and plane), (3) cordate to rounded leaf blade base (vs. cuneate), (4) concolorous to rarely discolorous leaf blade (vs. always discolorous), (5) leaf margins with teeth throughout (vs. above the base for c. 1.7-2 mm), (6) inflorescences with many flowers ( $v s$. few flowers), (7) spatulate petals (vs. oblanceolate). A more detailed list of diagnostic characters can be found in Table 1 .

Saunders (1995, unpublished) noticed differences between the two species (e.g. leaf blade color, calyx lobe length, anther morphology in brevistylous flowers, seed apex color) and proposed to treat $W$. brachypetala and $W$. ferruginea as distinct. In the same work, she also presented the illustration by Schumann (1886) in Flora Brasiliensis as being $W$. brachypetala. However, this illustration erroneously refers to $W$. ferruginea, being part of the protologue by Saint-Hilaire (1825).

Waltheria brachypetala belongs to Waltheria sect. Waltheria (as Euwaltheria) sensu Schumann (1886). Saunders (1995, unpublished) proposes another infrageneric classification for Waltheria, including W. brachypetala under W. subg. Pringlei. Saunders (1995, unpublished) also informally named this group "Waltheria ferruginea alliance", including species with stalked trichomes, mainly found on campo rupestre habitats.

Selected specimens examined-BRAZIL. Alagoas: Maceió, 2002, R.A. Silva 1796 (MAC!). Traipu, Serra das Mãos, 15 May 1990, R.P. Lyra-Lemos \& G.L. Esteves 2526 
Table 1. Main diagnostic features of the "Waltheria ferruginea alliance" sensu Saunders (1995, unpublished), comparing Waltheria biribiriensis, $W$. brachypetala, $W$. ferruginea and W. terminans.

\begin{tabular}{|c|c|c|c|c|}
\hline Character & W. biribiriensis & W. brachypetala & W. ferruginea & W. terminans \\
\hline Stipule length (mm) & c. 6.0 & $7.0-11.0$ & $3.0-3.2$ & $7.0-9.0$ \\
\hline Leaf blade length $(\mathrm{cm})$ & $3.5-13.0$ & $4.2-10.2$ & $3.4-10.4$ & $1.6-2.6$ \\
\hline Leaf blade shape & $\begin{array}{l}\text { Narrowly elliptic } \\
\text { to elliptic or lanceolate }\end{array}$ & Lanceolate & Elliptic & $\begin{array}{l}\text { Elliptic to oblong-elliptic } \\
\text { or lanceolate }\end{array}$ \\
\hline Leaf blade base & Subcordate to cordate & Cordate to rounded & Cuneate & Rounded to subcordate \\
\hline Leaf surfaces & Discolorous & $\begin{array}{l}\text { Concolorous, rarely } \\
\text { discolorous }\end{array}$ & Always discolorous & $\begin{array}{l}\text { Concolorous } \\
\text { to discolorous }\end{array}$ \\
\hline $\begin{array}{l}\text { Leaf blade margin } \\
\text { (when dry) }\end{array}$ & Plane & Plane & Slightly involute & Plane \\
\hline $\begin{array}{l}\text { Leaf margin tooth } \\
\text { arrangement }\end{array}$ & Throughout & Throughout & Above the base & Throughout \\
\hline Tooth dimensions (mm) & $2.0-2.2 \times 0.5-0.7$ & $0.8-1.8 \times 1.5-2.0$ & $0.4-0.7 \times 1.3-2.0$ & $0.3-0.9 \times 1.0-1.5$ \\
\hline No. of secondary vein pairs & $8-11$ & $14-17$ & $7-11$ & $7-8$ \\
\hline Inflorescence position & Along the branches & Along the branches & Along the branches & $\begin{array}{l}\text { In terminal portion } \\
\text { of the branches }\end{array}$ \\
\hline Peduncle length $(\mathrm{cm})$ & c. 0.2 & $0.3-2.2$ & $0.4-0.6$ & $0.5-1.0$ \\
\hline Inflorescence & Many-flowered & Many-flowered & Few-flowered & Few-flowered \\
\hline Bracteole shape & Oblong to lanceolate & Elliptic to widely elliptic & Elliptic to widely elliptic & $\begin{array}{c}\text { Oblanceolate to widely } \\
\text { obovate }\end{array}$ \\
\hline Fusion of the bracteoles & $\begin{array}{l}\text { Distinct or basally fused } \\
\text { for c. } 1.5 \mathrm{~mm}\end{array}$ & $\begin{array}{c}\text { Distinct or basally fused for } \\
1.8-2.2 \mathrm{~mm}\end{array}$ & Distinct & $\begin{array}{l}\text { Distinct or basally } \\
\text { fused for } 0.4-2.0 \mathrm{~mm}\end{array}$ \\
\hline Petals shape & Spatulate & Spatulate & Oblanceolate & Spatulate \\
\hline Shape of petal apex & Rounded to emarginate & Emarginate & Rounded & Rounded \\
\hline
\end{tabular}

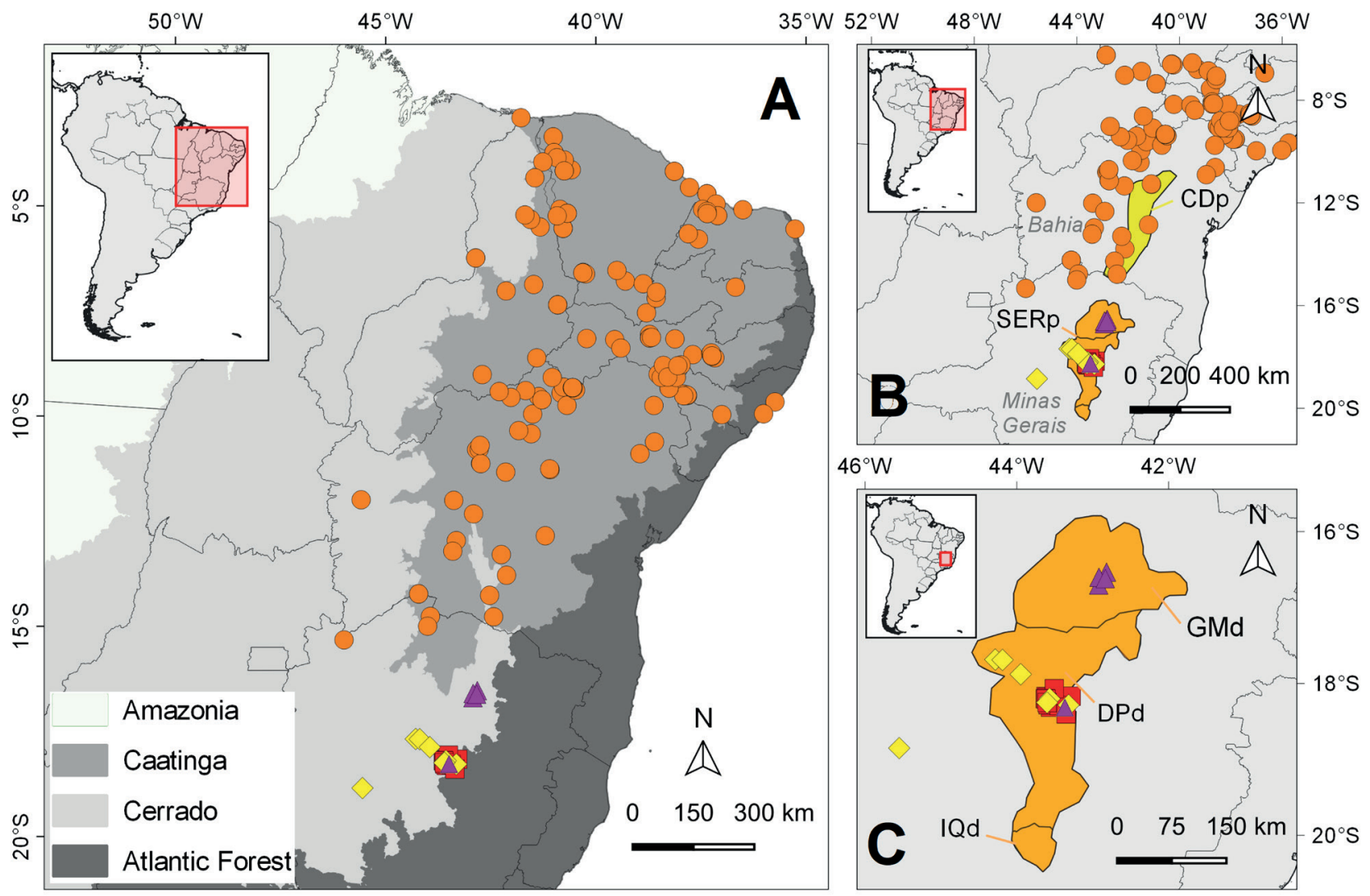

\section{- W. biribiriensis $-W$. brachypetala $\Delta$ W. ferruginea $\diamond W$. terminans}

Figure 1. Geographical distribution of Waltheria biribiriensis, W. brachypetala, W. ferruginea and W. terminans in eastern Brazil, with details of (A) phytogeographic domains and (B-C) bioregion delimitations. CDp: Chapada Diamantina Province; SERp: Southern Espinhaço Province, with its districts (GMd: Grão-Mogol District, DPd: Diamantina Plateau District and IQd: Iron Quadrangle District). 
(MAC!). Bahia: Andaraí, Rio Paraguaçu, 19 June 1984, G. Hatschbach \& R. Kummrow 48064 (SPF!). Bom Jesus da Lapa, rodovia para Santa Maria da Vitória, 05 April 1992, G. Hatschbach et al. 56608 (F [photo]!, MBM!). Casa Nova, Sítio Recanto, 9³6’49.18” S, 41¹6’44,77” W, 16 February 2008, J.A. Siqueira-Filho 1947 (HVASF!, MAC!). Feira da Mata, 14¹3'54" S, 44¹2'45” W, 06 May 2007, M.L. Guedes et al. 13302 (ALCB [not seen], MBM!). Morro do Chapéu, Lagedo Bordado, $11^{\circ} 15^{\prime} 31^{\prime \prime}$ S, 4105'43” W, 05 May 2007, J.M. Gonçalves et al. 217 (SPF!). Oliveira dos Brejinhos, Serra da Água Quente, 12¹9'46” S, 4254'24” W, 15 November 2012, E.L.M. Assis et al. 1087 (RB!). Xique-Xique, 1052' S, $42^{\circ} 45^{\prime}$ W, 17 March 1990, J.G. Saunders \& A.M. de Carvalho 3119 (CTES!). Ceará: Aracati, 17 June 1976, A. Fernandes s.n. (EAC 2800!). Crateús, Reserva Particular do Patrimônio Natural Serra das Almas, 06 June 2002, F.S. Araújo 1582 (EAC!). Granja, Distrito de Santa Terezinha, 03²1'30" S, 4100’39” W, 22 April 2015, E.B. Souza et al. 3384 (HUVA!). Tianguá, entre Tianguá e Viçosa do Ceará, 30 May 1979, A. Nunes s.n. (EAC 6226!). Minas Gerais: Manga, Gleba C-3, 30 April 1991, L.V. Costa et al. s.n. (BHCB 2243!). São João das Missões, $15^{\circ} 00^{\prime}$ S, 4400' W, 20 January 2010, C. Vidal 836 (BHCB!). Paraíba: Monte Horebe, 07¹1'41.30” S, 38³5'51.07”' W, 23 August 2012, R.A. Silva 2188 (HVASF!). Pernambuco: Buíque, Parque Nacional do Catimbau, 24 March 2018, T.S. Coutinho et al. 334 (UFP!). Ibimirim, Lagoa de Areia, 23 July 1994, A.M. Miranda et al. 1940 (HST!, RB!, UFP!). Petrolina, Campus da UNIVASF, 08 July 2019, T.S. Coutinho 457 (UFP!). Piauí: Amarante, Lage, 03 March 2005, A.M. Miranda et al. 4973 (ASE [photo]!, EAC!, HST!, HUEFS [photo]!, MAC!). Castelo do Piauí, ao lado da PI, 0513' S, $41^{\circ} 41^{\prime}$ W, 31 March 2004, J.M. Costa et al. 153 (UEC!). Floriano, 15 March 2009, A. Santos et al. s.n. (HST 16554!). Parnaíba, Lagoa Portinho, 03 October 1973, D. Araújo et al. 454 (RB!). Rio Grande do Norte: Ceará-Mirim, Praia de Muriú, 04 July 2014, J.S. Carvalho-Júnior \& L.A. Cestaro 36 (UFRN!). Mossoró, rodovia para Tibau do Sul, 0507'51" S, 37²1'05” W, 17 July 2013, J. Jardim \& A. Roque 6464 (RB!, UFRN!).

2. Waltheria ferruginea A. St-Hil., Fl. Bras. Merid. (quarto ed.) 1(4): 150. 1825. Type: BRAZIL. Minas Gerais: 'crescit in rupidus prope vicum Tapanhoacanga, haud longè ab urb Villa do Principe', 1816-1821, Saint-Hilaire s.n. (lectotype, designated here: P barcode P02273702 [photo]!; isolectotypes: MPU barcode MPU016436 [photo]!, MO [not seen], P02273703 [photo]!).

Shrubs 0.5-2.0 m tall. Branches terete, flattened at the apex, tomentose, trichomes stalked, multiradiate, ferrugineous, c. $0.5 \mathrm{~mm}$ long; bark dark brown, not rugose, somewhat resinous, not lenticellate. Stipules 3.0-3.2 ×0.3 $\mathrm{mm}$, linear, base truncate, apex acute, trichomes sessile, multiradiate, stellate and 2-radiate; veins inconspicuous. Leaves simple, alternate, distichally arranged throughout the branches; petiole $0.6-0.8 \times 0.12 \mathrm{~cm}$ long, slightly flattened dorso-ventrally, inconspicuously canaliculate, tomentose; leaf blade chartaceous, discolorous, 3.4-10.4 $\times 1.1-2.3(-3.5) \mathrm{cm}$, plane, elliptic, base cuneate, margin slightly involute when dried, finely serrate $1.7-2.0 \mathrm{~cm}$ above at the base, teeth $0.4-0.7 \times 1.3-2.0 \mathrm{~mm}$, apex acute, adaxial surface strigose, trichomes sessile, multirradiate, abaxial surface tomentose, trichomes stalked, multirradiate; venation actinodromous, 1-2 basal veins and 7-11 pairs of secondary veins, immersed adaxially, prominent abaxially. Inflorescence cymose, axillary, subsessile, few-flowered; bracts $5.0-8.0 \mathrm{~mm}$ long, 2-lobed; peduncle $0.4-0.6 \mathrm{~cm}$ long, tomentose. Flowers distylous, sessile, surrounded by four bracteoles; bracteoles 6.5-8.0 × 3.0-3.9 mm, distinct, elliptic to widely elliptic, apex acute, entire to 2-dentate, adaxial surface pubescent, abaxial surface tomentose, trichomes multiradiate on both surfaces, 1-3-nerved, obscured by trichomes. Calyx 5 -merous, gamosepalous, 6.2-8.0 × 2.7-3.0 mm, tubular-campanulate, 10-ribbed, externally tomentose, trichomes short-stalked, multiradiate, internally glabrous, tomentose on the lobes, trichomes stellate and sparse glandular trichomes, lobes 2.1-2.2 $\times$ $1.5-1.8 \mathrm{~mm}$, apex acuminate; nectary c. $0.5 \mathrm{~mm}$ long, on the base of the internal surface. Corolla 5-merous, dialypetalous, yellow, petals adnate to the staminal tube for c. $0.5 \mathrm{~mm}$, 5.0-6.1 × 1.2-1.5 mm, oblanceolate, both surfaces glabrous, apex rounded, glabrous. Stamens five, fully or partially connate forming a staminal tube, papillose apically, anthers dithecae, thecae parallel, dehiscence rimose; gynoecium apocarpous, carpel one and locule one, ovary obovoid, sericeous, trichomes simple and 2-3-radiate, style one, lateral, trichomes sessile, stellate, stigma one, elongateplumose. Longistylous form: stamens c. $4.2 \mathrm{~mm}$ long, staminal tube c. $3.0 \mathrm{~mm}$ long, free portion of the filaments c. $0.6 \mathrm{~mm}$ long, anthers c. $1.5 \mathrm{~mm}$ long, gynoecium c. 6.0 $\mathrm{mm}$ long, ovary $1.5 \times 0.7 \mathrm{~mm}$, style c. $4.0 \mathrm{~mm}$ long, stigma c. $1.0 \times 0.4 \mathrm{~mm}$ long. Brevistylous form: stamens c. $7.5 \mathrm{~mm}$ long, staminal tube c. 2.2-2.5 $\times 0.8 \mathrm{~mm}$ long, free portion of the filaments $3.6-3.8 \mathrm{~mm}$ long, anthers c. $1.1-1.2 \mathrm{~mm}$ long, gynoecium c. $3.5 \mathrm{~mm}$ long, ovary c. $1.1 \times 0.8 \mathrm{~mm}$, style c. $1.6 \mathrm{~mm}$ long, stigma c. $1.1 \times 0.3 \mathrm{~mm}$ long. Capsule one, c. $2.8 \times 1.8 \mathrm{~mm}$, obovoid, chartaceous at the apex, membranaceous below, apex rounded, pilose, trichomes stellate concentrated on the apex, dehiscence loculicidal; seed one, c. $2.0 \times 1.5 \mathrm{~mm}$, obovoid, black, glabrous, apex rounded.

Distribution and habitat-Waltheria ferruginea is endemic to the state of Minas Gerais. It can be found in elevations between 700-948 m in the Cerrado, associated to campo rupestre vegetation, specifically in the Southern Espinhaço Range province (specifically in the Grão-Mogol and Diamantina Plateau districts), where several other endemic plant species are reported (Colli-Silva et al. 2019) (Fig. 1).

Phenology - Flowers can be found in February, March, August and November and fruits in March. 
Conservation Status-According to our conservation assessment, Waltheria ferruginea is potentially classified as Endangered (EN) under the IUCN criterium B. This species is not found within protected areas and its Area of Occurrence is less than $500 \mathrm{~km}^{2}$.

Comments-Saunders (1995, unpublished) cites the herbarium $\mathrm{P}$ as the possible collection where the Waltheria ferruginea holotype is deposited; however, she did not verify or locate such material. We found two specimens of $W$. ferruginea labeled as Saint-Hilaire s.n. at P (barcodes P02273703 and P02273702), which warrants a lectotypification. We therefore chose the specimen P02273702 (barcode number) as the lectotype, as it possibly is the sample used for the illustration originally published by Saint-Hilaire. Morphological comments about distinctive characters between Waltheria ferruginea and W. brachypetala are discussed above and in Table 1.

Waltheria ferruginea belongs to $W$. sect. Waltheria (as Euwaltheria) according Schumann (1886), and to the informal W. subg. Pringlei and Waltheria ferruginea alliance sensu Saunders (1995, unpublished) (see previous description).

Additional specimens examined-BRAZIL. Minas Gerais: Botumirim, c. $12 \mathrm{Km}$ de Botumirim em direção à Adão Colares, 12 March 1999, A. Rapini \& M.L. Kawasaki 760 (SPF!, UFP!). Cristália, Bem Querer, 10 November 1991, G. Hatschbach et al. 54991 (MBM!). Diamantina, $15 \mathrm{Km}$

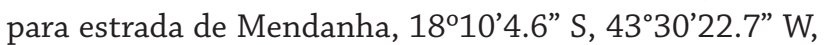
22 February 2003, G.O. Romão et al. 942 (ESA!, RB!). GrãoMogol, 16³2' S, 4249' W, 13 August 1989, A. Freire-Fierros et al. s.n. (BHCB 88955!, CTES 188027!, K 1213095 [photo]!, SPF 67939!); idem, Vale do Rio Itacambiruçu, 26 February 1986, T.B. Cavalcanti et al. s.n. (SPF 42952!, UEC 45885!); idem, 16³7' S, 4250' W, 28 March 1990, J.G. Saunders et al. 3179 (MBM!, NY 2342594, 813681 [photos]!).

3. Waltheria biribiriensis J.G. Saunders ex T.S. Coutinho \& Colli-Silva sp. nov.

Type: BRAZIL. Minas Gerais: Diamantina, "estrada para Biribiri, c. 4 km para Biribiri”, 18¹0'13.3” S 4336'53.8” W, 950 m elev., 23 January 2007, J.R. Pirani, M.F.A. Calió, B.P.F. Loeuille and E.G. Martins 5689 (holotype: SPF!; isotypes: SP!, UFP!). (Fig. 2).

It resembles Waltheria ferruginea by its stalked and multiradiate trichomes on branches and leaves and axillary inflorescences, but differs by its whitish to slightly yellowish trichomes (vs. ferrugineous), bracteoles usually 2-3-lobed to rarely entire apically ( $v s$. always entire apically), and by the capsules chartaceous at apex and membranaceous below (vs. capsules chartaceous throughout).

Shrubs 0.7-2.0 m tall. Branches erect, terete, flat towards the apex, puberulent, glabrescent, trichomes stalked, multiradiate, whitish to slightly yellowish, c. $0.4 \mathrm{~mm}$ long; bark reddish-brown when dry, rugose longitudinally, not resinous, lenticellate. Stipules c. 6.0 $\times 1.0 \mathrm{~mm}$, linear, base truncate, apex acute, trichomes similar to the branches, 1-nervate, hidden by the dense trichome layer. Leaves simple, alternate, distichally arranged throughout the branches; petiole 0.4-1.3 $\times 0.1-0.2 \mathrm{~cm}$, angular, not canaliculate, pubescent to canescent; leaf blade chartaceous, strongly discolorous, 3.5-13.0 × 1.3-6.5 cm, narrowly elliptic to elliptic or lanceolate, base subcordate to cordate, margin plane, finely serrate, teeth $2.0-2.2 \times$ $0.5-0.7 \mathrm{~mm}$, apex acute, both surfaces densely pilose, trichomes stalked, multiradiate; venation actinodromous, 8-11 pairs of secondary veins and 3 basal veins, immersed adaxially, prominent abaxially. Inflorescence cymose, axillary along the branches, sessile to subsessile, many-flowered; bracts 3-lobed, 7.0-8.2 $\mathrm{mm}$ long; peduncle c. $0.2 \mathrm{~cm}$ long, densely pubescent. Flowers distylous, sessile; bracteoles 4 , $8.0 \times 1.5-1.8 \mathrm{~mm}$, distinct or basally fused for c. $1.5 \mathrm{~mm}$, oblong to lanceolate, apex acute, 2-3-lobed to rarely entire, adaxial surface tomentose, trichomes sessile, stellate, abaxial surface scabrous, trichomes short-stalked, multiradiate, 2-4 nervate. Calyx 5-merous, gamosepalous, 5.0-7.0 $\times$ 2.8-3.2 mm, tubular-campanulate, 10-ribbed, externally scabrous, trichomes short-stalked to stalked, multiradiate, internally glabrous, tomentose on the lobes, lobes 1.0-2.2 $\times 1.0-1.2 \mathrm{~mm}$, apex acute to subacute; nectary c. $0.7 \mathrm{~mm}$ long, on the base of the internal surface. Corolla 5-merous, dialypetalous, yellow, petals adnate to the staminal tube for c. $0.4 \mathrm{~mm}, 6.0-6.5 \times 1.5-2.0 \mathrm{~mm}$, spatulate, both surfaces glabrous, apex rounded to emarginate, glabrous. Stamens five, partially connate forming a staminal tube, papillose apically, anthers dithecae, thecae parallel, dehiscence rimose; gynoecium apocarpous, carpel one and locule one, ovary obovoid, sericeous, trichomes simple and 2-3-radiate, style one, lateral, tomentose, trichomes sessile, stellate, stigma one, clavate. Longistylous form: stamens c. $4.0 \mathrm{~mm}$ long, staminal tube c. $2.5 \times 1.0 \mathrm{~mm}$ long, free portion of the filaments c. $0.8 \mathrm{~mm}$ long, anthers c. $1.3 \mathrm{~mm}$ long, gynoecium c. $7.1 \mathrm{~mm}$ long, ovary c. $1.5 \times 0.9 \mathrm{~mm}$, style c. $5.5 \mathrm{~mm}$ long, stigma c. $1.0 \times 0.2 \mathrm{~mm}$. Brevistylous form: stamens 5.0-6.0 mm long, staminal tube $2.0-2.1 \times 1.0 \mathrm{~mm}$, free portion of the filaments $2.0-2.2 \mathrm{~mm}$ long, anthers $1.4-1.8$ $\mathrm{mm}$ long, gynoecium $4.0-5.2 \mathrm{~mm}$ long, ovary $1.3-1.5 \times$ $1.0 \mathrm{~mm}$, style $2.0-2.1 \mathrm{~mm}$ long, stigma $0.5-0.6 \times 0.2 \mathrm{~mm}$. Capsule one, 3.0-3.4 × 1.5-2.0 mm, obovoid, chartaceous on the apex, membranaceous below, apex rounded, hirsute, stellate trichomes concentrated on the apex, dehiscence loculicidal; seed one, 2.2-2.5 ×1.3-1.5 mm, obovoid, brown to dark brown, glabrous, apex not crenulate.

Distribution and habitat-Waltheria biribiriensis is endemic to the state of Minas Gerais, growing on campo rupestre vegetation in the Cerrado, in elevations between 800-1200 m, in the Southern Espinhaço province (Diamantina Plateau district), a bioregion that concentrates a significant amount of endemic plant species (Colli-Silva et al. 2019) (Fig. 1). 


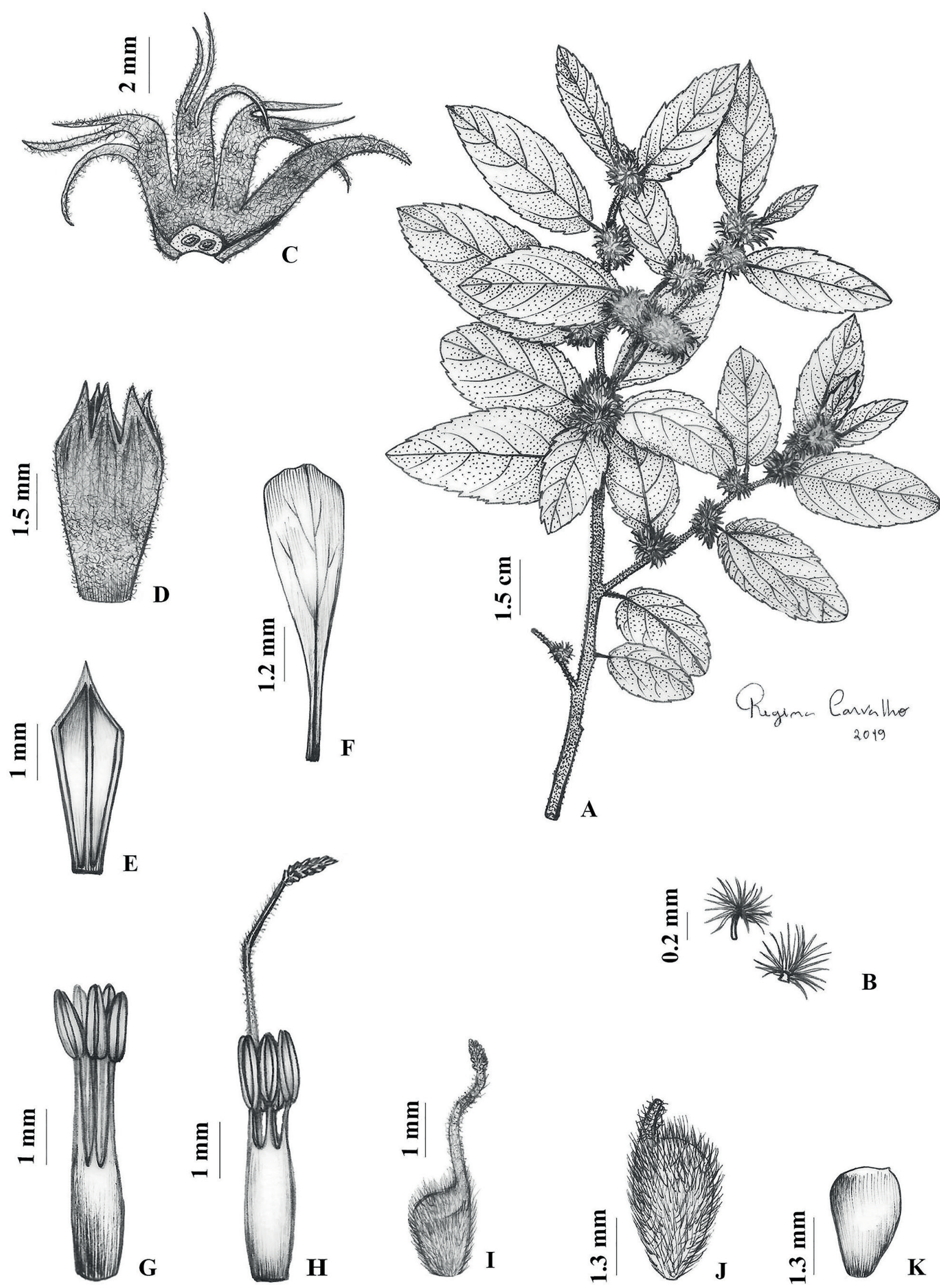

Figure 2. Waltheria biribiriensis J.G. Saunders ex T.S. Coutinho \& Colli-Silva. A. Flowering branch. B. Stalked and short-stalked multiradiate trichomes. C. Detail of bracteole with two flowers removed. D. Calyx. E. Internal view of the calyx lobe with trichomes removed. F. Petal. G. Stamens of brevistylous flowers. H. Stamens of longistylous flowers, highlighting part of style and stigma. I. Gynoecium of brevistylous flowers. J. Capsule. K. Seed. L. Gynoecium. M. Capsule. N. Seed. (A-G, I: J.R. Pirani et al. 5689; H: M.M. Arbo et al. 5028; J-K: N. Hensold et al. 3103). 
Etymology-The specific epithet refers to "Biribiri", a district in the municipality of Diamantina, Minas Gerais, where the type specimen was collected.

Phenology-Flowering specimens are reported from December to May, with fruits in May.

Conservation status-According to the conservation assessment under IUCN criterium B, Waltheria biribiriensis is potentially Vulnerable (VU). Waltheria biribiriensis has not yet been recorded within protected areas and its Area of Occurrence is less than $500 \mathrm{~km}^{2}$.

Comments - The name Waltheria biribiriensis was firstly described by Saunders (1995, unpublished), but never effectively published. Some vouchers of $W$. biribiriensis have been determined as $W$. ferruginea, as both are shrubs with stalked multirradiate trichomes on their branches and leaves. Nevertheless, Waltheria biribiriensis can be morphologically distinguished from $W$. ferruginea by the following characters: whitish to yellowish trichomes (vs. ferruginous trichomes), leaf blade 3.5-13.0 × 1.3-6.5 $\mathrm{cm}$ (vs. up to $6.3 \times 2.2 \mathrm{~cm}$ ), cordate to subcordate base (vs. cuneate), oblong to lanceolate bracteoles (vs. elliptic to widely elliptic), bracteoles apically 2-3-lobed to rarely entire (vs. not 3-lobed), spatulate petals (vs. oblanceolate). Further diagnostic characters are presented in Table 1.

Waltheria ferruginea and W. biribiriensis are both endemic to the campo rupestre vegetation in Minas Gerais, but the latter has a more restricted distribution (Fig. 1). Some populations of the two species are found in sympatry; however, they can be easily separated by the previously mentioned morphological characters (Tab. 1). Additionally, Waltheria biribiriensis is found in elevations up to $1200 \mathrm{~m}$, while Waltheria ferruginea is restricted to elevations around $900 \mathrm{~m}$.

Waltheria biribiriensis belongs to $W$. sect. Waltheria (as Euwaltheria) according to Schumann (1886), and to the informal W. subg. Pringlei and Waltheria ferruginea alliance sensu Saunders (1995, unpublished).

Additional specimens examined (Paratypes) BRAZIL. Minas Gerais: Diamantina, estrada para Biribiri, 18¹0' S, 4337' W, 08 May 1982, N. Hensold et al. s.n. (MBM 193570!, SPF 23311!); estrada para povoado de Três Barras, $3 \mathrm{~km}$ de Diamantina, 15 May 1987, V.L. Scatena s.n. (RB 341682!, SPF 47251!); estrada para Milho Verde, Km 9, 18¹8'41" S, 4333'19” W, 19 May 2018, J.N. Nakajima et al. 4807 (SP!); margem da estrada para Curralinho, próximo a gruta do Salitre, $18^{\circ} 16^{\prime} 45^{\prime \prime}$ S, 4332'02” W, 21 May 2016, J.E.Q. Faria 5862 (RB!, SP!); Mirante do Cruzeiro, $18^{\circ} 13^{\prime} 40^{\prime \prime}$ S, 433' $22^{\prime \prime}$ W, 09 April 2016, J.E.Q. Faria 5616 (RB!, SP!); Rio dos Cristais, 25 March 1966, A.P. Duarte 9648 (RB!); 6 km N de Diamantina, caminho a Biribiri, c. $18^{\circ} 11^{\prime}$ S , $43^{\circ} 36^{\prime}$ W, 14 February 1991, M.M. Arbo et al. 5028 (SPF!); 5 $\mathrm{km}$ from road to Diamantina on road to Biribiri, $18^{\circ} 08^{\prime}$ S, 4340’ W, 26 March 1990, J.G. Saunders et al. 3167 (BHCB!, F [photo]!, K [3 sheets; photos]!, MBM [2 sheets]!,
NY [2 sheets; photos]!); road Diamantina-Biribiri, 08 December 1997, R.C. Forzza et al. 510 (SPF!); Ponte do Acaba Mundo, sobre o rio Jequitinhonha, c. $17 \mathrm{~km}$ E de Extração (Curralinho), base da Serra do Gavião, 18¹7'25" S, 43²6'21" W, 08 February 2009, M.S. Ferrucci et al. 2856 (SPF!); Serra do Espinhaço at Lapinha, c. $19 \mathrm{Km} \mathrm{N}$ of Serro on road to Diamantina, 24 February 1968, H.S. Irwin et al. 20799 (MBM!). c. $20 \mathrm{Km}$ E to Diamantina, near Rio Jequiti, 15 March 1970, H.S. Irwin et al. 27546 (CTES!, MBM!, NY [photo]!, RB!); estrada para Biribiri, 08 December 1992, H.F. Leitão Filho et al. 27508 (ESA!, RB!, UEC!). Serro, base do Pico do Itambé, 05 May 1942, G.M. Magalhães 2088 (BHCB!).

4. Waltheria terminans J.G. Saunders ex T.S. Coutinho \& Colli-Silva sp. nov.

Type: BRAZIL. Minas Gerais: Joaquim Felício, Serra do Cabral, Bocaina, 23 Nov 1984, M.C.H. Mamede, A.M. Giulietti, R.M. Harley \& B. Stannard s.n. (holotype: SPF 35981!; isotypes: K barcode K1217181 \& 1217182 [photos]!, UFP 86645!). (Fig. 3).

Waltheria terminans resembles $W$. brachypetala by its shrubby lifeform and stalked multiradiate trichomes on the branches, but differs by having spirally arranged leaves (vs. distichally arranged), leaf blade with 7-8 pairs of secondary veins ( $v s .14-17$ pairs of secondary veins), and axillary inflorescences only in the terminal portion of the branches (vs. axillary along all the branch).

Shrubs 0.7-3.0 m tall. Branches erect, terete, flat upward at apex, scabrous, trichomes stalked, multiradiate, yellowish, c. $0.3 \mathrm{~mm}$ long; bark brownish when dry, neither rugose nor resinous, lenticels on older branches. Stipules 7.0-9.0 $\times 0.6 \mathrm{~mm}$, linear, base truncate, apex acute, trichomes sessile, multiradiate, 1 -nervate, hidden by the dense trichome layer. Leaves simple, alternate, spirally arranged throughout the branches; petiole $0.2-$ $0.3 \times 0.2 \mathrm{~cm}$, angular, not canaliculate, canescent; leaf blade chartaceous, discolorous, 1.6-2.6 × 0.9-1.4 cm, elliptic to oblong-elliptic or lanceolate, base rounded to subcordate, margin inconspicuously serrate, teeth $0.3-0.9 \times 1.0-1.5 \mathrm{~mm}$, apex acute, rarely rounded, both surfaces scabrous, trichomes stellate, more abundant on the abaxial surface; venation actinodromous, 2 basal veins and 7-8 pairs of secondary veins, immersed adaxially, prominent abaxially. Inflorescence cymose, axillary in terminal portions of the branches, pedunculate, fewflowered; bracts 2-3-lobed, 7.0-8.2 mm long; peduncle $0.5-1.0 \mathrm{~cm}$ long, scabrous. Flowers distylous, sessile, surrounded by four bracteoles; bracteoles 7.0-10.5 $\times$ 2.1-4.0 mm, distinct or basally fused for $0.4-2.0 \mathrm{~mm}$, oblanceolate to widely obovate, apex acute to rounded, entire to 2-3-dentate apically, adaxial surface pubescent, trichomes stellate and sessile, abaxial surface scabrous, trichomes multiradiate and short-stalked, 6-7 nervate. Calyx 5-merous, gamosepalous, 5.0-7.0 × 1.8-3.1 mm, 


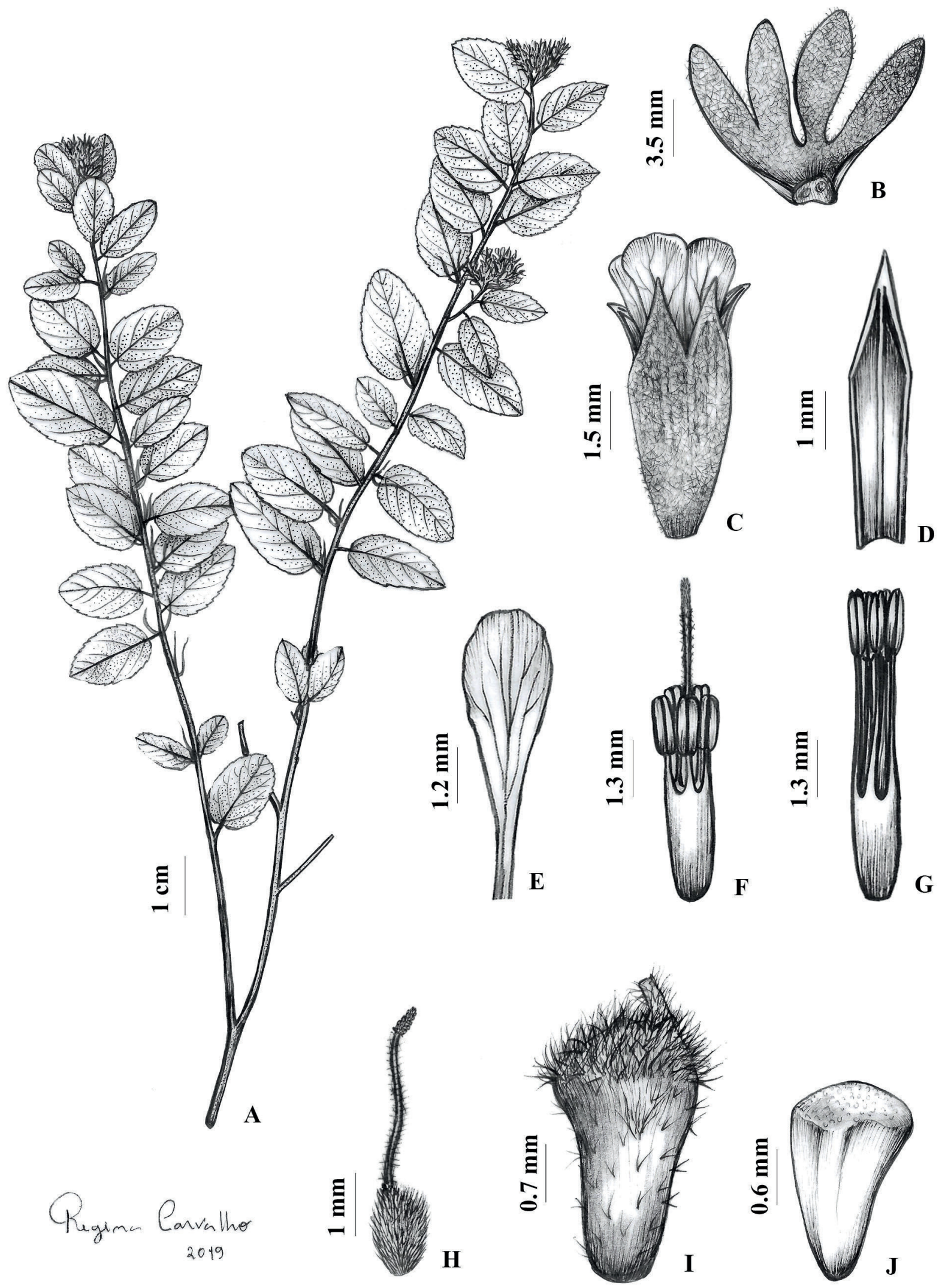

Figure 3. Waltheria terminans J.G. Saunders ex T.S. Coutinho \& Colli-Silva. A. Flowering branch. B. Detail of the bracteoles with two flowers removed. C. Flower. D. Internal view of the calyx lobe with trichomes removed. E. Petal. F. Stamens of brevistylous flowers. G. Stamens of longistylous flowers. H. Gynoecium of brevistylous flowers. I. Capsule. J. Seed. (A-E, G-H: M.C.H. Mamede et al. s.n. [SPF 35981]; F: A. Rapini et al. 477; I-J: B. Stannard et al. 3103). 
tubular-campanulate, 10-ribbed, externally scabrous, trichomes multiradiate, sessile, internally glabrous, tomentose on the free portion of the lobes, lobes up to c. $2.2 \times 1 \mathrm{~mm}$, apex acuminate; nectary c. $0.6 \mathrm{~mm}$ long, on the base of the internal surface. Corolla 5-merous, dialypetalous, yellow, petals adnate to the staminal tube for $0.4-0.8 \mathrm{~mm}, 5.5-6.5 \times 1.8-2.0 \mathrm{~mm}$, spatulate, both surfaces glabrous, apex rounded, glabrous. Stamens five, partially connate into a staminal tube, papillose apically, anthers dithecae, thecae parallels, dehiscence rimose; gynoecium apocarpous, carpel one and locule one, ovary obovoid, sericeous, trichomes simple and 2-radiate, style one, lateral, tomentose, trichomes sessile, stellate, stigma one, filiform. Longistylous form: stamens $4.2-5.0 \mathrm{~mm}$ long, staminal tube $2.2-2.8 \times 1.0 \mathrm{~mm}$ long, free portion of the filaments c. $0.8-1.0 \mathrm{~mm}$ long, anthers $1.4-1.5$ $\mathrm{mm}$ long, gynoecium $5.0-6.0 \mathrm{~mm}$ long, ovary $1.2-1.8 \times$ $0.6 \mathrm{~mm}$, style $3.0-4.3 \mathrm{~mm}$ long, stigma c. $0.8 \times 0.1 \mathrm{~mm}$. Brevistylous form: stamens $6.2-6.5 \mathrm{~mm}$ long, staminal tube $2.0-2.5 \times 1.0 \mathrm{~mm}$, free portion of the filaments 3.2-3.5 mm long, anthers 1.4-1.5 mm long, pistil 4.3-5.5 mm long, ovary $1.2-1.5 \times 0.9 \mathrm{~mm}$, style $2.5-3.5 \mathrm{~mm}$ long, tortuous near the apex, stigma $0.6-0.8 \times 0.1 \mathrm{~mm}$. Capsule one, c. $3.3 \times 1.8 \mathrm{~mm}$, obdeltate, chartaceous at the apex, then membranaceous elsewhere, apex truncate-rounded, entirely hirsute, trichomes stellate, concentrated on the apex, dehiscence loculicidal; seed one, c. $2.0 \times 1.2 \mathrm{~mm}$, obovoid, dark brown, glabrous, apex crenulate, rounded.

Distribution and habitat-Known only from the state of Minas Gerais, occurring in some municipalities and localities of campo rupestre vegetation in the Southern Espinhaço province (Diamantina Plateau district), in elevations between 1100-1300 m (Fig. 1).

Etymology-The specific epithet refers to the location of the inflorescences, which, in addition to being axillary, are always arranged in the terminal portions.

Phenology-Waltheria terminans is found with flowers between November and January to May and fruits in November.

Conservation status-According to our preliminary conservation assessment, Waltheria terminans, similarly to W. biribiriensis, is potentially Vulnerable (VU). Only one population of Waltheria terminans has been registered within a protected area, in Serra do Cabral State Park. Its Area of Occurrence is less than $500 \mathrm{~km}^{2}$.

Comments-Waltheria terminans was previously described by Saunders (1995, unpublished) as a new taxon but not effectively published. It can be distinguished from its relative species, $W$. brachypetala, by the spiral leaf arrangement (vs. distichous in $W$. brachypetala), petiole 0.2-0.3 cm long (vs. 0.6-1.1 cm long), leaf blade 1.6-2.6 $\mathrm{cm}$ long (vs. 4.3-9.5 cm long), inflorescences only in the terminal portions (vs. along the branches), oblanceolate to widely obovate bracteoles (vs. elliptic to widely elliptic) and petals rounded at the apex (vs. emarginate). Further morphological characters are summarized in Table 1.

Some populations of $W$. terminans are found in sympatry with $W$. biribiriensis and $W$. ferruginea, where they share the same lifeform; however, $W$. terminans can be easily distinguished by its leaves $1.6-2.6 \mathrm{~cm}$ long ( $v s$. $3.4-13.0 \mathrm{~cm}$ long) and by other characters summarized in Table 1. Waltheria terminans reaches the highest elevations when compared to other species above, up to $1,300 \mathrm{~m}$.

Waltheria terminans belongs to $W$. sect. Waltheria (as Euwaltheria) according to Schumann (1886), and to the informal $W$. subg. Pringlei and Waltheria ferruginea alliance sensu Saunders (1995, unpublished).

Additional specimens examined (Paratypes) BRAZIL. Minas Gerais: Buenópolis, Cachoeira de Curimataí, 14 November 2010, E. Barbosa et al. 2906 (MBM!); Joaquim Felício, Bocaína, 22 November 1984, B. Stannard et al. s.n. (K barcode K001217140 [photo]!, SPF 35919!); Serra do Cabral, 18 January 1996, G. Hatschbach et al. 64342 (MBM!); Diamantina, 20-26 km WSW de Diamantina, caminho a Conselheiro Mata, MG-220, $18^{\circ} 17^{\prime}$ S, 4349' W, 18 May 1990, M.M. Arbo et al. 4380 (SPF!); idem, 02 April 1992, P. Schwake 8333 (RB!); 8 km NE Diamantina, caminho a Mendanha, córrego Soberbo, $18^{\circ} 12^{\prime}$ S, 433'' W, 14 February 1991, M.M. Arbo 5086 et al. (CTES!, SPF!). Lassance, Serra do Cabral, $17^{\circ} 41^{\prime} \mathrm{S}$, 4417' W, 24 March 2000, J.R. Pirani et al. 4634 (SPF!).

\section{Key to the species of the informal Waltheria ferruginea alliance sensu Saunders (1995, unpublished) (based on our own studies)}

1. Leaf blade $\leq 2.6 \mathrm{~cm}$ long; inflorescences in the terminal portion of the branches; stigma filiform .......... W. terminans

1 '. Leaf blade $\geq 3.4 \mathrm{~cm}$ long; inflorescences along the branches; stigma elongate-plumose or clavate ...........................2

2. Leaf blade base cuneate; inflorescences few-flowered; oblanceolate petals

W. ferruginea

2'. Leaf blade base subcordate to cordate or rounded to cordate; inflorescences many-flowered; spatulate petals ..................... 3

3. Stipules up to $6.0 \mathrm{~mm}$ long; leaf blade with 8-11 pairs of secondary veins; oblong to lanceolate bracteoles, apically

2-3-lobed or rarely entire W. biribiriensis

3'. Stipules exceeding $7.0 \mathrm{~mm}$ long; leaf blade with 14-17 pairs of secondary veins; elliptic to widely elliptic bracteoles, entire to 2-3-dentate apically . W. brachypetala 


\section{Acknowledgements}

We thank the following Brazilian funding institutions: CAPES (Coordenação de Aperfeiçoamento de Pessoal de Nivel Superior - Finance Code 001) for maintaining the post-graduation programs in which the first two authors are enrolled; FAPESP (Fundação de Amparo à Pesquisa do Estado de São Paulo, Grant ID 2017/19295-1) and CNPq (Conselho Nacional de Desenvolvimento Científico e Tecnológico, Grant ID 141327/2017-0) for funding the researchers. We are also grateful to Regina Carvalho for the illustrations; and to Carolina Siniscalchi for the review of the English.

\section{References}

Alverson WS, Whitlock BA, Nyffeler R, Bayer C, Baum D. 1999. Phylogeny of the core Malvales: evidence from $n d h F$ sequence data. American Journal of Botany 86: 1474-1486.

Bayer C, Kubitzki K. 2005. Malvaceae. In: Kubitzki K. (ed.) The families and genera of vascular plants. Vol. V. Heidelberg, Springer-Verlag. p. 225-311.

Colli-Silva M, Vasconcelos NTC, Pirani JR. 2019. Outstanding plant endemism levels strongly support the recognition of campo rupestre provinces in mountaintops of eastern South America. Journal of Biogeography 46: 1723-1733.

Coutinho TS, Alves M. 2019. A new distylous Waltheria L. (Byttnerioideae, Malvaceae) from the state of Bahia, Brazil. Systematic Botany 44: 681-685.

Coutinho TS, Alves M. 2020. Waltheria glabribracteata (Byttnerioideae, Malvaceae), a new species with elongate-plumose stigmas from South America. Phytotaxa 430: 294-299.

Cristóbal CL. 1968. Estudio morfológico de los granos de polen de Byttneria (Sterculiaceae). Pollen Spores 10: 57-72.

Dauby G, Stévart T, Droissart V, et al. 2017. ConR: An R package to assist large-scale multispecies preliminary conservation assessment using distribution data. Ecology and Evolution 7: 11292-11303.

Esteves G. 2010. Waltheria. In: Forzza RC, Baumgratz JFA, Bicudo CEM, et al. (eds.) Catálogo de plantas e fungos do Brasil. Vol. II. Rio de Janeiro, Andrea Jakobsson Estúdio. p. 1225-1226.

Harris JG, Harris MW. 2001. Plant identification terminology - an illustrated glossary. Payson, Spring Lake Publishing.

Kohler E. 1976. Pollen dimorphism and heterostyly in the genus Waltheria L. (Sterculiaceae). In: Ferguson IK, Muller I. (eds.) The evolutionary significance of the exine. London, Linnean Society of London by Academic Press. p. 147-161
R Development Core Team. 2017. R: A language and environment for statistical computing. R Foundation for Statistical Computing, Vienna, Austria. https://www.R-project.org/. 01 Mar. 2020.

Radford AE, Dickison WC, Massey JR, Bell CR. 1974. Vascular plant systematics. New York, University of North Carolina.

Richardson JE, Whitlock BA, Meerow AW, Madriñán S. 2015. The age of chocolate: diversification history of Theobroma and Malvaceae. Frontier in Ecology and Evolution 3: 120. doi: 10.3389/ fevo.2015.00120

Rose JN. 1899. A synopsis of the North American species of Waltheria. Contributions of the United States National Herbarium 5: 183-185.

Saint-Hilaire AF. 1825. Flora Brasiliae Meridionalis 1. Amsterdam, Netherlands, Parisiis Apud A. Belin Bibliopolam.

Saunders JG, Pozner R. 2007. A new penicillate-stigma species of Waltheria (Sterculiaceae, Hermannieae) endemic to Belize. Novon 17: 79-86.

Saunders JG. 1993. Four new distylous species of Waltheria (Sterculiaceae) and a key to the Mexican and central American species and species groups. Systematic Botany 18: 356-376.

Saunders JG. 1995. Systematics and Evolution of Waltheria (Sterculiaceae-Hermannieae). PhD Thesis, The University of Texas, Austin. [unpublished].

Saunders JG. 2005. New species of Waltheria (Hermannieae, Byttnerioideae, Malvaceae) from Paraguay, Argentina, and Venezuela, and two news records for Paraguay. Darwiniana 43: 201-211.

Schumann K. 1886. Waltheria. In: Martius CFP, Eichler AW, Urban I. (eds.) Flora Brasiliensis, 12(3). Monachii, Leipzig, Friedrich Fleischer in Comm. p. 50-68

Silva-Coutinho T, Marin-Pérez L, Alves M. 2019. Primer registro de Waltheria glomerata (Malvaceae) para Brasil. Revista Mexicana de Biodiversidad 90: e902821. doi: 10.22201/ ib.20078706e.2019.90.2821

Thiers B. 2019 [continuously updated]. Index herbariorum: A global directory of public herbaria and associated staff. New York, New York Botanical Garden's virtual herbarium. http://sweetgum.nybg. org/science/ih/. 20 Dec. 2019.

Turczaninow NS. 1858. Secundam partem herbarii Turczaninowiani, nunc universitati caesareae charcowiensis. Bulletin de la Société Impériale des Naturalistes de Moscou 31: 213-216.

Weberling F. 1992. Morphology of flowers and inflorescences. Cambridge, Cambridge University Press.

Webster GL, Del-Arco-Aguilar MJ, Smith BA. 1996. Systematic distribution of foliar trichome types in Croton (Euphorbiaceae). Botanical Journal of the Linnean Society 121: 41-57.

Whitlock BA, Bayer C, Baum DA. 2011. Phylogenetic relationships and floral evolution of the Byttnerioideae ("Sterculiaceae" or Malvaceae s.l.) on sequences of the chloroplast gene, $n d h F$. Systematic Botany 26: $420-437$. 